Dome C Astronomy and Astrophysics Meeting

M. Giard, F. Casoli and F. Paletou (eds)

EAS Publications Series, 14 (2005) 87-92

\title{
PRECISION CMB POLARIZATION FROM DOME-C: THE BRAIN EXPERIMENT
}

S. Masi ${ }^{1}$, P. de Bernardis ${ }^{1}$, C. Giordano ${ }^{1}$, F. Nati ${ }^{1}$, F. Piacentini ${ }^{1}$, G. Polenta ${ }^{1}$, M. Veneziani ${ }^{1}$, M. Gervasi ${ }^{2}$, G. Sironi ${ }^{2}$, A. Tartari ${ }^{2}$, M. Zannoni ${ }^{2}$, S. Peterzen ${ }^{3}$, J. Bartlett ${ }^{4}$, Y. Giraud-Heraud ${ }^{4}$, M. Piat ${ }^{4}$, C. Rosset ${ }^{4}$, M. Giard ${ }^{5}$, R. Pons ${ }^{5}$, B. Maffei ${ }^{6}$, P. Ade ${ }^{6}$, W. Gear ${ }^{6}$, P. Mauskopf ${ }^{6}$, L. Piccirillo ${ }^{6}$, G. Pisano ${ }^{6}$ and G. Savini ${ }^{6}$

\begin{abstract}
In the current cosmological scenario, part of the linearly polarized emission of the CMB is expected to be rotational ( $B$-modes). This component is due to tensor perturbations of the metric produced by primordial gravitational waves, which are generated a split-second after the Big Bang. The signal expected is of the order of $\lesssim 0.1 \mu \mathrm{K}$, well below the non-rotational component of the polarization signal ( $E$-modes), and beyond the sensitivity of present generation instruments. New, more sensitive instruments are developed in several labs, with the goal to measure the $B$-modes. Control of systematics and foregrounds will be the key to make the results of these experiments believable. In this paper we shortly outline BRAIN, a bolometric interferometer devoted to $B$-modes research, and its pathfinder experiment, devoted to test the Dome-C site.
\end{abstract}

\section{Introduction}

The polarization of the Cosmic Microwave Background (CMB) offers a unique opportunity to study the universe during its earliest moments, and examine a number of important questions in fundamental physics. Soon after the Big Bang, it's believed that the universe entered an accelerated expansion phase, known as

\footnotetext{
${ }^{1}$ Department of Physics, University of Rome "La Sapienza", Italy

2 Department of Physics, University of Milano Bicocca, Italy

${ }^{3}$ ISTARS and Agenzia Spaziale Italiana, Italy

${ }^{4}$ Cosmology Group, Collège de France, Paris, France

${ }^{5}$ CESR, Toulouse, France

${ }^{6}$ Department of Physics and Astronomy, University of Cardiff, UK
} 
cosmic inflation, which lead to the creation of cosmological density perturbations, as well as primordial gravitational waves. The former are currently studied in detail by CMB anisotropy experiments; the latter have yet to be discovered - and CMB polarization is the key. Gravity waves create a unique signature in CMB polarization, $B$-modes (rotational), distinguished from the signal produced by ordinary density perturbations, which only produce $E$-modes (irrotational) (see e.g. Zaldarriaga \& Seldjak 1998). The amplitude of the $B$-mode component is proportional to the square of the inflationary energy scale, and so can be directly measured. This is of outmost importance for both Cosmology and Fundamental Physics, testing Physics at energies close to the Grand Unification Scale $\left(\sim 10^{16} \mathrm{GeV}\right)$, much higher than those produced by any foreseeable particle accelerator. A different source of $B$-mode polarization is the gravitational lensing of the CMB $E$-mode signal by large-scale structures forming along the line-of-sight. This offers a tool to study the evolution of these structures, which touches in turn on fundamental physics issues like the nature of dark energy and neutrino masses. The expected level of the $B$-mode signal is very low, about 100 times smaller than the CMB temperature anisotropy (see e.g. Wu 2002; Kaplinghat et al. 2003; Smith et al. 2004). This is a real challenge to experimentalists, who can win only by means of a coordinated approach involving independent experiments, using orthogonal advanced technologies, and located at exceptional sites.

\section{The BRAIN Instrument}

BRAIN (Background RAdiation INterferometer) is aimed to be one of these experiments. BRAIN is a bolometric interferometer, combining the high sensitivity of bolometric detectors and the robustness of interferometric experiments (see e.g. Piccirillo 2004).

The hope is that despite of the different systematic effects affecting interferometers and imagers, any detection of $B$-modes is finally consistently obtained by experiments of both classes, thus enhancing the confidence in the detection of such a small signal. In other words, we hope that even for the elusive CMB $B$-modes we will finally arrive to the same situation we have now with CMB anisotropy, where directly comparable anisotropy maps have been detected by bolometric experiments (BOOMERanG, MAXIMA, Archeops) and by radiometers (DASI, WMAP) (de Bernardis et al. 2004; Abroe et al. 2004; Hamilton et al. 2004; Wright 2002; Bennett et al. 2004).

This effort needs the very best site on the Earth for microwave astronomy, so we plan to carry out the measurement from the French-Italian CONCORDIA station in Antarctica (123 $23^{\prime} 42^{\prime \prime} \mathrm{E}, 70^{\circ} 06^{\prime} 06^{\prime \prime} \mathrm{S}, 3233 \mathrm{~m}$ asl). This is well suited, due to the exceptionally low and stable temperature and humidity in the Antarctic winter (see e.g. Dall' Oglio \& Valenziano 1999; and Storey 2004).

The working principle of an interferometer is well known: observing a point source in the sky, with two telescopes (antennas) separated by a distance $D$, the electromagnetic waves collected by the telescopes will have a difference of phase. Combining optically the two EM waves and then detecting the resulting signal will 
produce a Young interference pattern, depending on the direction of the source observed. This pattern is closely related to the Fourier Transform of the sky brightness. In BRAIN, for each circular antenna we separate the two orthogonal polarizations, using high efficiency ortho-mode transducers, and then we correlate orthogonal polarizations coming from different antennas through $90^{\circ}$ hybrids. The mixed signals are phase-modulated and recombined (using additional $90^{\circ}$ hybrids) in different bolometric detectors. The signals from the detectors are synchronously demodulated and combined to estimate the Stokes parameters. A sketch of a single baseline, employing two circular antennas and four bolometers is shown in (Fig. 1). From this configuration all Stokes parameters of polarization can be retrieved. The

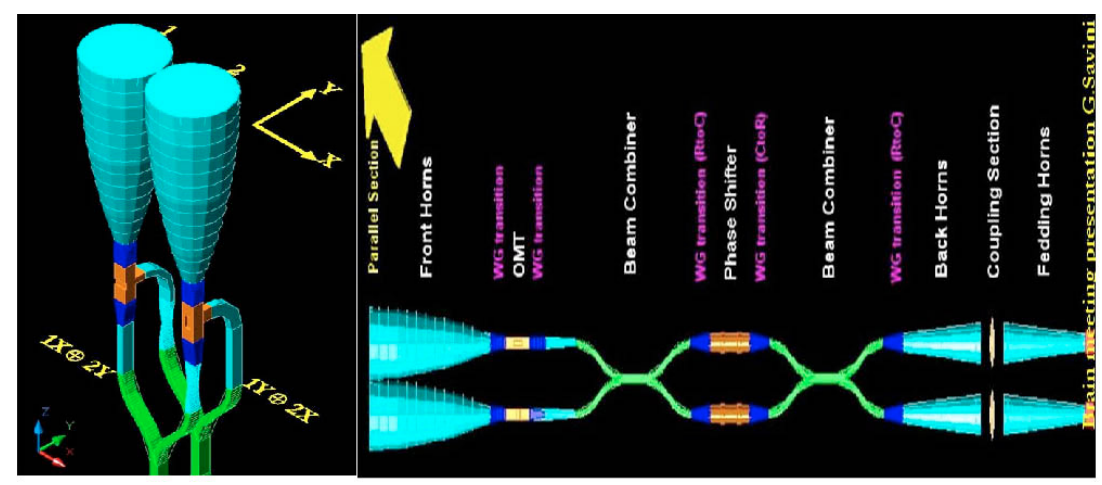

Fig. 1. Block-diagram of one baseline of the BRAIN interferometer.

final instrument - still being optimized - will integrate a compact array of about hundred antennas. While most of the microwave components needed are basically off-the-shelf at $90 \mathrm{GHz}$ and $150 \mathrm{GHz}$, some of them have to be developed and optimized. Among these the most critical is the cryogenic phase modulator. We are still comparing different options for it, including slab-type operated by piezo actuators.

\section{The BRAIN Pathfinder}

This is a complex experiment, and its implementation in Dome-C needs to be as smooth as possible. We have thus planned to build a pathfinder experiment to demonstrate the automated operation of a $0.3 \mathrm{~K}$ cryogenic system in Dome-C during the Antarctic winter, and to study the microwave polarized emission of the atmosphere and of the Galaxy during winter observations from Dome-C. We designed an automated cryogenic system operating without cryogenic liquids: due to the extreme environmental conditions $\left(\sim-60^{\circ} \mathrm{C}\right)$ and the high cost of Helium storage, Helium refilling during the Antarctic winter is nearly impossible. The solution we have selected is based on a pulse tube (PT) cryocooler, modified to work in the Antarctic environment. The cryocooler (Fig. 2) is able to reach a 
stable minimum temperature between $2 \mathrm{~K}$ and $4 \mathrm{~K}$, depending on the heat load produced by the cold optics. To cool the detectors down to $0.3 \mathrm{~K}$ we use a double stage ${ }^{4} \mathrm{He} /{ }^{3} \mathrm{He}$ refrigerator (Fig. 2). The compressor, radiator, and all the elec-

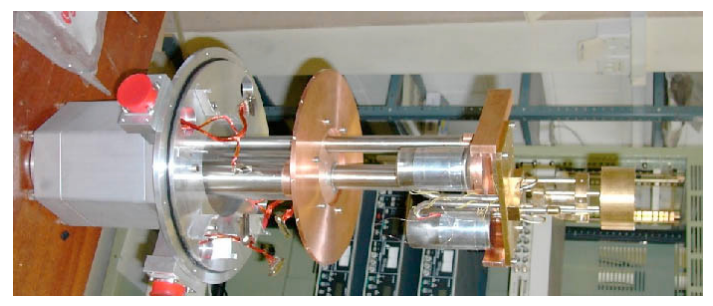

Fig. 2. "Dry" cryogenic system composed of a Pulse Tube cooler and a ${ }^{3} \mathrm{He} /{ }^{4} \mathrm{He}$ self contained refrigerator. A similar system, optimized for Antarctic operation, will be used for BRAIN. All shields have been removed. During operation, the four flanges visible from left to right are at $300-260 \mathrm{~K}, 30 \mathrm{~K}, 2.5 \mathrm{~K}, 0.27 \mathrm{~K}$ respectively.

tronics of the system are contained in an insulated shelter. A power control unit monitors the temperature of all the relevant parts of the system as well as the pressure inside the dewar and the availability of the external supply. The power control is able to operate the heaters and the windows of the shelter to maintain a stable operation temperature inside. In case of failure of the external supply, the controller operates the correct restarting sequence when power becomes available again. The core of the control system is a very low-power micro-controller able to operate autonomously for about one year from a standard laptop battery. It is enclosed in an insulated vessel and kept within its operating temperature range. The control system is also programmed to manage the vacuum system and to recycle the ${ }^{4} \mathrm{He} /{ }^{3} \mathrm{He}$ refrigerator, which is designed to keep the bolometers at $0.3 \mathrm{~K}$ for $\sim 90$ hours. One of the main problems when operating with pulse tube coolers is the low level of vibrations induced from the rotary valve, which produces helium pulses, approximately 1 per sec. This results in microphonic noise centered at $1 \mathrm{~Hz}$, but extending up to $1 \mathrm{kHz}$ due to the hiss of $\mathrm{He}$ gas entering the tube. This is critical when operating with high impedance composite bolometers, while is supposed to be a minor issue for TES bolometers. In order to minimize the coupling of the PT cold heat to the sensors, we decided to mount both the cold optics and the detectors on the external dewar shell, while the PT is mechanically floating with respect to the dewar shell. All the thermal links between the different PT stages and the experiment are made using flexible copper braids.

The unpolarized emission of the atmosphere is one of the main issues for ground-based experiments. For millimeter observation, there are windows available at 90 and $150 \mathrm{GHz}$, while higher frequency channels can be used to monitor the residual contamination. In addition there is a polarized component. The Earth magnetic field acts on spin 1 oxygen molecules producing Zeeman splitting. For the line at $118.8 \mathrm{GHz}$, the Zeeman splitting is $\sim \pm 1 \mathrm{MHz}$, depending on the 


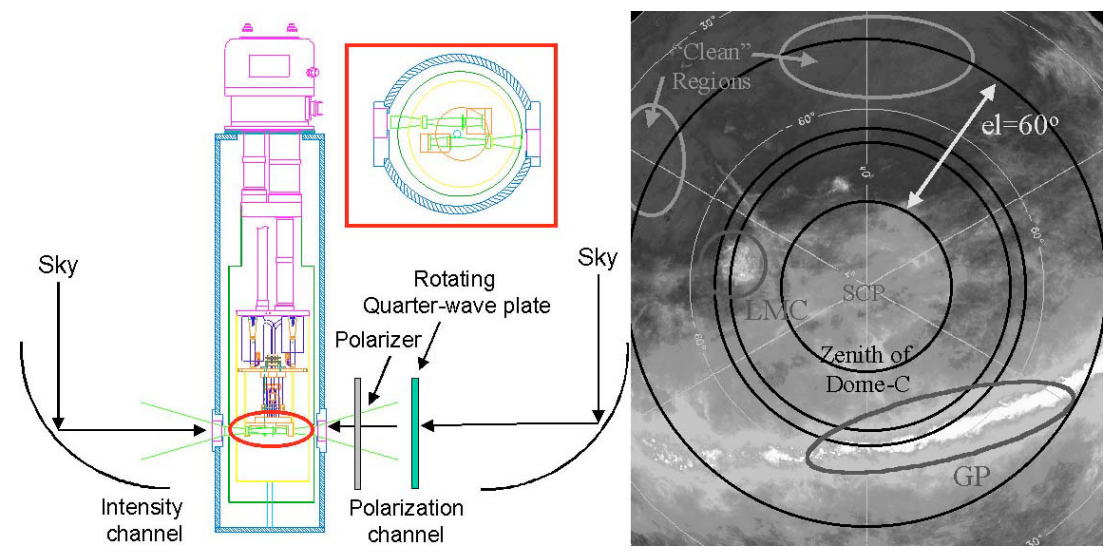

Fig. 3. Left: schematic configuration of the BRAIN pathfinder. Right: observable sky from CONCORDIA (IRAS $100 \mu \mathrm{m}$ image centered on the SCP). The inner circle represents the path of the CONCORDIA zenith, the other circles represent the border of the regions observable from Dome-C at elevations of $\sim 73^{\circ}, \sim 70^{\circ}, \sim 60^{\circ}$.

strength of the magnetic field. The relative contribution of the three lines depends on the angle between the direction of the magnetic field and the line of sight of our observation. The split lines corresponding to quantum number $m= \pm 1$ along the line of sight parallel to the magnetic field are fully circularly polarized. There are no measures of this effect for the microwave emission of the atmosphere. However, order of magnitude calculations suggest that at $143 \mathrm{GHz}$ (in the far wings of the lines) the amplitude of the linearly and circularly polarized components are respectively $10^{-9}$ and $10^{-6}$ of the total intensity (Hanany \& Rosenkranz 2003). The CMB polarization has only a linear component, and, in principle, we should not be worried by the polarization of the atmosphere. But in a real experiment there are many spurious effects which could convert the polarization from circular to linear, like e.g. the presence of a non ideal mirror. The final effect on the CMB measurements will depend strongly on the inhomogeneity of the magnetic field and on the scan strategy of the experiment, and it is likely to be reduced significantly. One of the scientific purposes of the BRAIN pathfinder is then to measure the circularly polarized emission of the atmosphere at $143 \mathrm{GHz}$. This is made simply using a rotating quarter-wave plate (QWP) and a polarizer in front of a bolometric detector horn, as in Figure 3. The QWP converts circular to linear polarization and vice versa with high efficiency. The quarter-wave plate is $1.6 \mathrm{~mm}$ thick sapphire disk with a useful diameter of $60 \mathrm{~mm}$. The rotating mount is equipped with a Superior Electric step motor and gears which allow a maximum modulation frequency of $10 \mathrm{~Hz}$ and a life duration of more than one year. The polarizer is composed of etched $A l$ wires and is put besides a $0.666 \mathrm{~mm}$ thick polypropylene vacuum window. The horns are the same used for the Archeops 
$143 \mathrm{GHz}$ channels and are cooled down to $4 \mathrm{~K}$. The output of the detector for this configuration is:

$$
w=\frac{1}{2}\left[I+Q \frac{1+\cos (4 \omega t)}{2}+U \frac{\sin (4 \omega t)}{2}+V \sin (2 \omega t)\right]
$$

where $I, Q, U$ and $V$ are the four Stokes parameters and $\omega$ is the frequency of the mechanical rotation of the QWP. So the total intensity $I$ and part of the $Q$ parameter are DC signals, and are filtered out by means of a high-pass filter. The linear polarization signal $U$ and the remaining part of $Q$ will be demodulated as $4 \omega$ components, and finally the circular polarization $V$ as a $2 \omega$ component. The intensity $I$ is measured using another bolometer in a standard photometer configuration. The system will be able to scan the Antarctic sky by means of an alt-az mount making constant elevation scans. In addition to local storage, selected data are transmitted to European labs through IRIDIUM. Readily observable at high elevation from Dome-C are the Galactic Plane, the Magellanic Clouds, the "BOOMERanG" clean region (Masi et al. 2001) at high Galactic latitudes. In one year of observations with 6 months in the dark, the instrument will be able to produce polarization maps of all the regions above.

\section{Conclusions}

Bolometric interferometry is a promising technique. As implemented in BRAIN, it will produce high-sensitivity measurements of the $B$-modes power spectrum of the $\mathrm{CMB}$, the hot topic of cosmological investigation nowadays. The location of Dome-C is expected to be exceptionally good, and will be experimentally tested by a pathfinder experiment, starting in 2005, aimed to measure the Stokes parameters of atmospheric and Galactic emission. This work is supported in Italy by the Programma Nazionale di Ricerche in Antartide and by MIUR.

\section{References}

Abroe, M.E., et al. 2004, ApJ, 605, 607

Bennett, C.L., et al. 2004, ApJS, 148, 1

Dall' Oglio, G. \& Valenziano, L. 1999, PASA, 16, 167

de Bernardis, P., et al. 2004, in IAU Symposium, 216, MAPS OF THE COSMOS, eds.

M. Colless \& L. Staveley-Smith, ASP, 2004, astro-ph/0311396

Hamilton, J.-Ch. \& Benoit, A. 2004, the Archeops Collaboration, astro-ph/0310788

Hanany, S. \& Rosenkranz, P. 2003, astro-ph/0307052

Hu, W. 2002, Phys. Rev. D, 65, 023003

Kaplinghat, et al. 2003, Phys. Rev. Lett., 91, 241301

Masi, S., et al. 2001, ApJ, 553, L93

Piccirillo, L. 2004, Mem.S.A.It.Suppl., 2, 200

Smith, et al. 2004, astro-ph/0402442

Storey, J., et al. 2004, this volume

Wright, E.L. 2002, http://www.astro.ucla.edu/ wright/BO0Mdat.html

Zaldarriaga, M. \& Seldjak, U. 1998, Phys. Rev. D, 58, 023003 